SILVA, T.A. et al. Prolapso de cervix, vagina e útero em vacas - Revisão de Literatura. PUBVET, Londrina, V. 5, N. 27, Ed. 174, Art. 1176, 2011.

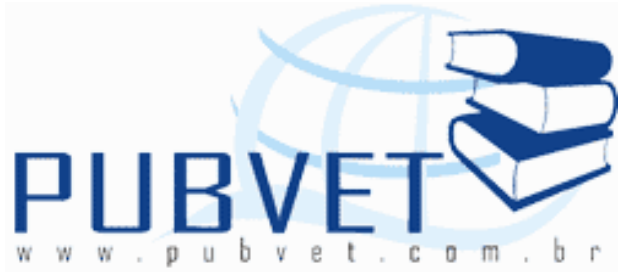

PUBVET, Publicações em Medicina Veterinária e Zootecnia.

\title{
Prolapso de cervix, vagina e útero em vacas - Revisão de Literatura
}

Thiago Alexandre da Silva ${ }^{1 *}$, Rafael Rocha de Souza ${ }^{2}$, Mariela Silva Moura ${ }^{3}$, Francisco Sales Resende Carvalho ${ }^{4}$

${ }^{1}$ Médico Veterinário

${ }^{2}$ Residente em Patologia Clínica da Faculdade de Medicina Veterinária, Universidade Federal de Uberlândia. Uberlândia, MG.

${ }^{3}$ Mestranda em Ciências Veterinárias pela Faculdade de Medicina Veterinária, Universidade Federal de Uberlândia, MG.

${ }^{4}$ Docente da Faculdade de Medicina Veterinária, Universidade Federal de Uberlândia. Uberlândia, MG.

*Autor para correspondência: Rua Ceará, s/n, Bloco 2D, CEP 38400-902, Uberlândia, MG, Brasil. E-mail: thiagoads85@hotmail.com

\section{Resumo}

Os prolapsos de vagina, cervix e útero são responsáveis por grandes perdas econômicas e desequilíbrio na eficiência reprodutiva de bovinos de leite e corte (BARTOLOMEU et al 1997; MARQUES et al 1991). Pandit et al. (1982) citou que, a maioria dos animais tratados, com reposição mecânica dos órgãos e sutura de vulva, se tornaram estéreis após prolapso vaginal/ uterino, mostrando cervicites crônicas, com índice de reincidência da afecção em $34,88 \%$ dos animais. As causas dos prolapsos, em todas as suas variações, permanecem de certo modo indefinidas, não se conhece as causas exatas. 
SILVA, T.A. et al. Prolapso de cervix, vagina e útero em vacas - Revisão de Literatura. PUBVET, Londrina, V. 5, N. 27, Ed. 174, Art. 1176, 2011.

Todavia, parecem estar correlacionadas com altas concentrações sericas de estrógeno, deficiência de alguns minerais, especialmente cálcio e fósforo, predisposição genética, má formação do canal obstétrico e edemas vulvares (ROBERTS, 1979). Segundo Shukla; Pareth (1987) a literatura relata uma incidência de prolapso de 3,35\% a 5,36\%, dependendo da raça explorada ou manejo realizado. Levando em conta o tamanho do rebanho nacional podemos começar a visualizar os prejuízos que essa patologia reprodutiva, em todas as suas variações, acarreta no montante total de produtividade das fêmeas do rebanho Brasileiro. Estudando e tendo uma compreensão melhor desta patologia reprodutiva podemos evitar problemas, encontrar soluções e minimizar perdas econômicas.

Palavras-chave: Prolapso vaginal, prolapso de cervix, prolapso uterino; vacas.

\section{Prolapse of cervix, vagina and uterus in cows - Literature Review}

\section{Abstract}

Prolapses of uterus, cervix and vagina are answerable for economic losses and unbalance on reproductive efficiency of dairy an beef cows (BARTOLOMEU et al 1997; MARQUES et al 1991). Pandit et al. (1992) cited that the most of treated animals with mechanic replacement of organs and suture of vulva, became sterile after prolapse vaginal/uterine, showing chronicle cervicitis, with recidivation index of $34,88 \%$ at this affection on animals. The causes of prolapses in all yours variations stands in certain manner undefined, precise causes aren't known. Nevertheless, seems to be correlated with high plasmatic estrogens concentration, mineral deficiency, essentially phosphorus and calcium, genetic predisposition, obstetric passage bad developed and vulva intumesce (ROBERTS, 1979). According to Shukla; Pareth (1987) the literature mention prolapse incidence of $3,35 \%$ until $5,36 \%$, based on explored race or management accomplished. Regarding how big is the national herd, we might begin to see the prejudice that this reproductive patology cause in all yours 
SILVA, T.A. et al. Prolapso de cervix, vagina e útero em vacas - Revisão de Literatura. PUBVET, Londrina, V. 5, N. 27, Ed. 174, Art. 1176, 2011.

variations on whole amount of productivity of cows in Brazilian herd. Studding and having a better understand of this reproductive patology we can avoid problems find solutions and minimize economic loses.

Keywords: Cervix prolapse, cows, prolapse, uterine prolapse, vaginal prolapse.

\section{Introdução}

O Brasil possuía no ano de 2004 uma população de 176,1 milhões de bovinos, 79,5\% de corte (ANUALPEC, 2004), e 80\% está composta de raças zebu ou cruzamentos de zebu (JOSAHKIAN, 2000). Em conseqüência, existe um grande número de sub-populações de vários tamanhos, com composição racial Bos indicus $\times$ Bos indicus e Bos indicus $\times$ Bos Taurus, as quais se enquadram na descrição de população multirracial.

No ano de 2005 essa população já era 175,1 milhões de cabeças, segundo dados do Instituto Brasileiro de Geografia e Estatística. O tamanho do rebanho brasileiro, segundo o Censo Agropecuário de 2006, divulgado no final de 2007, causou surpresa entre os envolvidos com a bovinocultura, a expectativa, segundo os dados oficiais, era de um rebanho de 205 milhões de cabeças em 2006. Em 21 de dezembro de 2007 foram divulgados, pelo IBGE, os dados preliminares do Censo Agropecuário, que tem por base o ano de 2006, o contingente de bovinos foi para 169,9 milhões de cabeças. (ANUALPEC, 2008) O setor da bovinocultura começou a ganhar contornos mais

precisos. Com tabulação ate 30 de novembro do ano de 2006, já estavam no banco de dados do órgão informações sobre 5,2 milhões de estabelecimentos rurais, 2.6 milhões dos quais com bovinos de leite ou corte. Faltavam responder ao censo apenas quatro mil proprietários, o que, apesar de representar menos de $1 \%$ do total, resultara em ajustes. 
SILVA, T.A. et al. Prolapso de cervix, vagina e útero em vacas - Revisão de Literatura. PUBVET, Londrina, V. 5, N. 27, Ed. 174, Art. 1176, 2011.

\section{Rebanho bovino por Regiäa*}

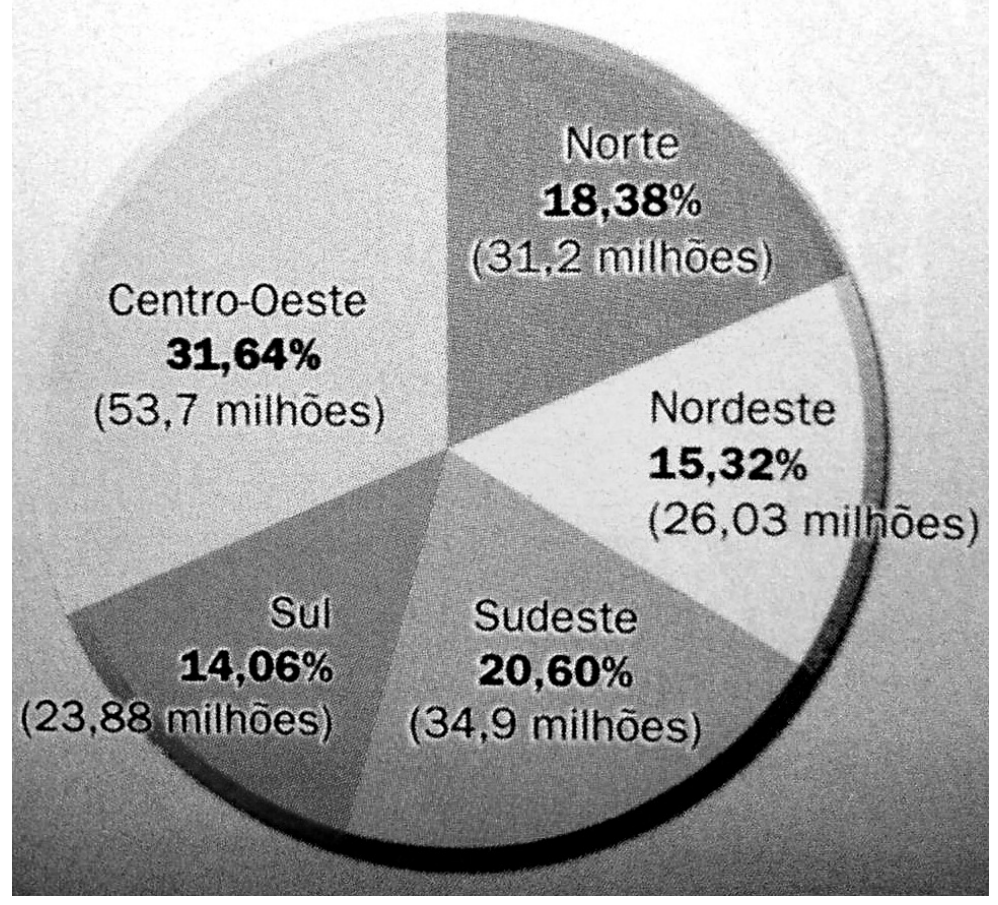

Figura 1: Censo agropecuário de 2006. Versão preliminar sujeita a alteração a partir de dados pendentes. IBGE (2006).

Fonte: Anuário DBO (2008)

Segundo Antonio Carlos Florido que é coordenador da gerencia técnica do Censo, o número de animais poderia chegar a 180 ou 183 milhões de animais. (ANUARIO DBO, 2008) Os dados mais recentes, referentes ao ano de 2007 sobre o contingente do rebanho bovino brasileiro, foram divulgados pelo ANUALPEC 2008, o tamanho do rebanho foi estimado em 167,5 milhões de cabeças. Há previsões para o ano de 2008 quando poderá ser atingido o número de 169,8 milhões de cabeças. (ANUALPEC, 2008) 
SILVA, T.A. et al. Prolapso de cervix, vagina e útero em vacas - Revisão de Literatura. PUBVET, Londrina, V. 5, N. 27, Ed. 174, Art. 1176, 2011.

Tabela 1: Balanço da bovinocultura no Brasil em milhões de cabeças, do ano de 2004 a 2008.

\begin{tabular}{cccccc}
\hline ANO & 2004 & 2005 & 2006 & 2007 & 2008 \\
\hline $\begin{array}{l}\text { REBANHO EM MILHÕ̃ES } \\
\text { DE CABEÇAS }\end{array}$ & 176,1 & 175,1 & 169,9 & 167,5 & $169,8^{*}$ \\
\hline
\end{tabular}

Fonte: ANUALPEC (2008)

Nota: *previsões para o ano de 2008.

Levando em conta o tamanho do rebanho nacional é possível começar a visualizar os prejuízos que essa patologia reprodutiva, em todas as suas variações, acarreta no montante total de produtividade das fêmeas do rebanho Brasileiro. Estudando e tendo uma compreensão melhor desta patologia reprodutiva podemos evitar problemas, encontrar soluções e minimizar perdas econômicas.

\section{Causas e tratamentos}

O prolapso do útero pode ocorrer em qualquer espécie; porém, ele é mais comum nas vacas leiteiras e nas porcas, menos freqüente nas ovelhas e raro nas éguas, nas cadelas e nas gatas. A etiologia é obscura e a ocorrência é esporádica (MANUAL MERCK DE VETERINARIA, 2006).

A administração de estilbestrol é conhecida como amolecedora dos ligamentos genitais pelo fato de aumentar 0 volume do trato genital (GEOFFREY, 1979).

Segundo o Manual Merck de Veterinária (2006) o decúbito com os quartos posteriores mais baixos que os quartos anteriores, a invaginação do útero, o excesso de tração para aliviar uma distocia e a hipocalcemia têm sido todos incriminados como causas contribuintes.

O prolapso do útero geralmente ocorre dentro de poucas horas após o parto, quando a cervix está aberta, o útero perdeu o tonus e os ligamentos uterinos encontram-se bastante distendidos. 
SILVA, T.A. et al. Prolapso de cervix, vagina e útero em vacas - Revisão de Literatura. PUBVET, Londrina, V. 5, N. 27, Ed. 174, Art. 1176, 2011.

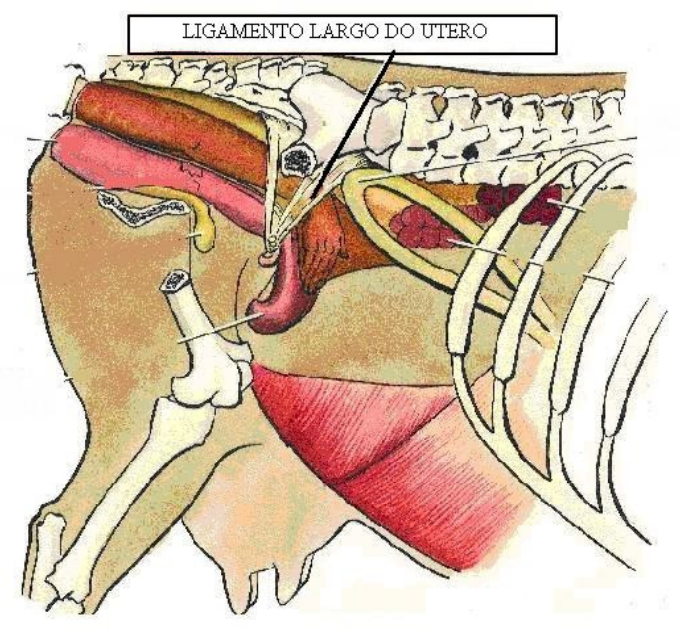

Figura 2: Esquema do ligamento largo do útero, no inicio da gestação de uma fêmea bovina.

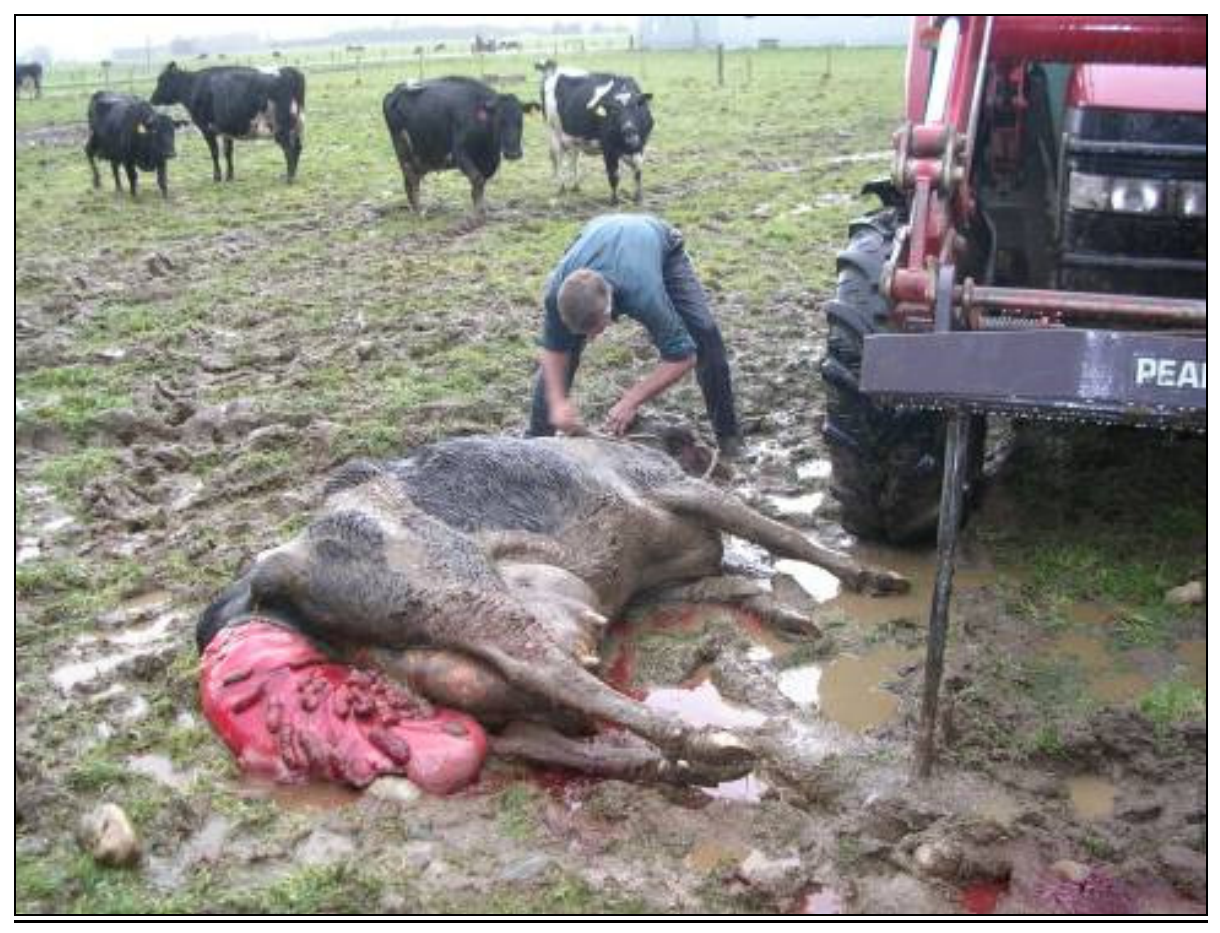

Figura 3: Prolapso uterino pós-parto em uma vaca da raça holandesa com sete anos de idade. 
SILVA, T.A. et al. Prolapso de cervix, vagina e útero em vacas - Revisão de Literatura. PUBVET, Londrina, V. 5, N. 27, Ed. 174, Art. 1176, 2011.

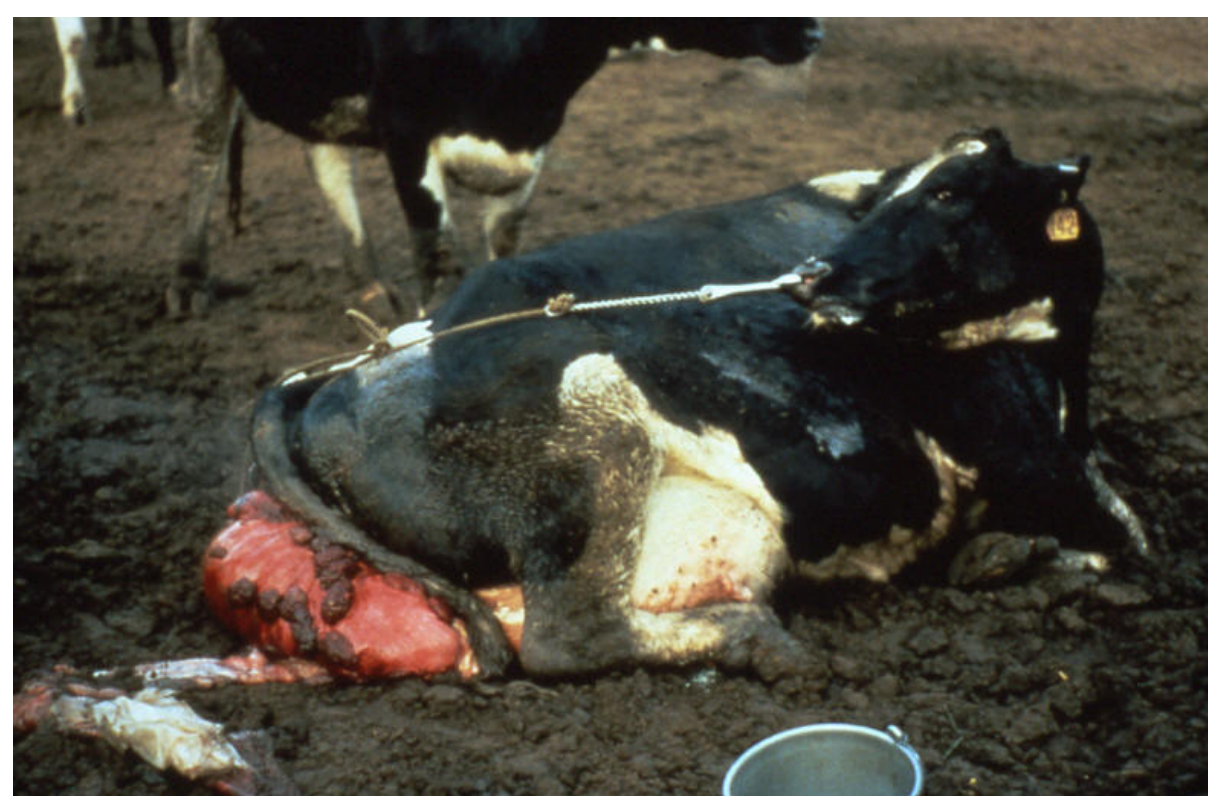

Figura 4: Prolapso uterino pós-parto em uma vaca holandesa com quatro anos de idade.

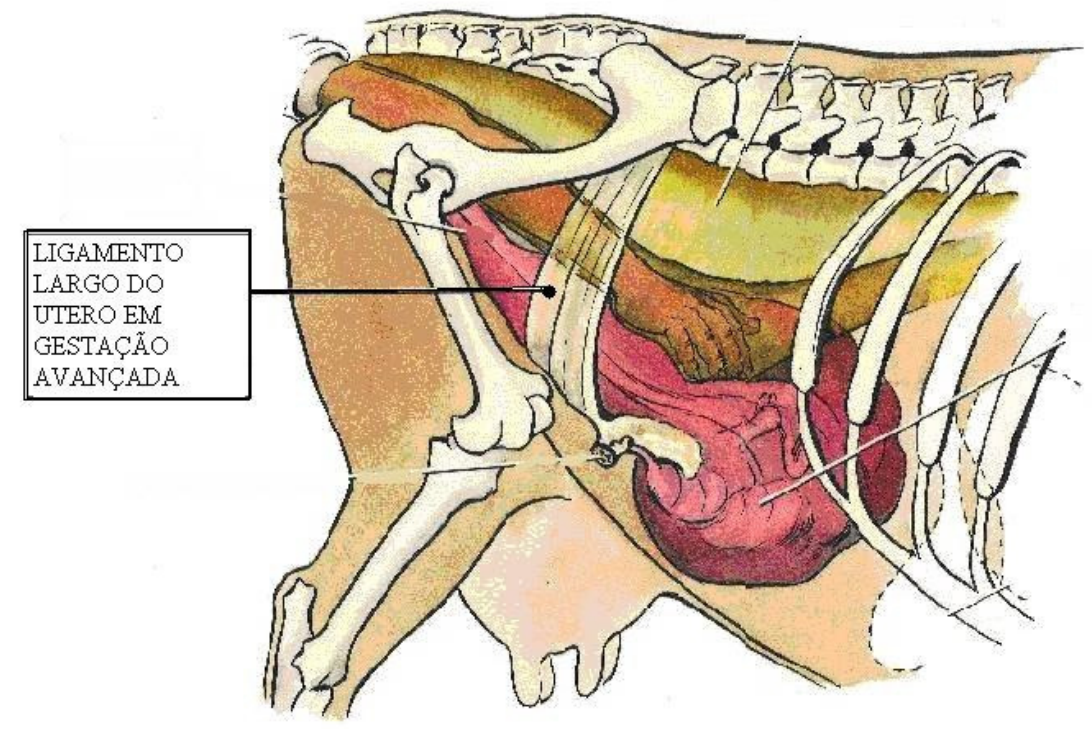

Figura 5: Esquema do ligamento largo do útero em gestação avançada de uma fêmea bovina. 
SILVA, T.A. et al. Prolapso de cervix, vagina e útero em vacas - Revisão de Literatura. PUBVET, Londrina, V. 5, N. 27, Ed. 174, Art. 1176, 2011.

O prolapso geralmente é completo e a massa do útero geralmente pende por baixo dos jarretes do animal afetado. Nas vacas, o tratamento envolve a remoção da placenta (se ainda estiver presa) e a limpeza completa da superfície endometrial. Retorna-se então o útero para a sua posição normal por um de vários métodos. Primeiro, deve-se administrar uma anestesia epidural. Se a vaca ficar de pé, deve-se limpar o útero, elevá-lo ao nível da vulva sobre uma bandeja (ou por meio de uma maca segura por dois assistentes) e então ao colocá-lo por meio da aplicação de uma pressão anterior firme, começando na porção cervical e progredindo gradualmente para o ápice.

Uma vez recolocado o útero, deve-se inserir a mão na extremidade de ambos os cornos uterinos para se certificar de que não haja uma invaginação remanescente. Se a vaca ficar em decúbito, deve-se posicioná-la com os quartos posteriores elevados para movimentá-la para uma área inclinada ou para colocá-la em decúbito esternal com as patas traseiras estendidas para trás. (MANUAL MERCK DE VETERINARIA, 2006).

Um método alternativo envolve a elevação dos quartos posteriores com algum tipo de elevador preso às patas anteriores, colocando assim a vaca em decúbito dorsal. Recoloca-se o útero como indicado anteriormente.

Nas porcas e nos pequenos animais, pode-se conseguir a reposição por meio da manipulação simultânea do útero, a partir do exterior com uma mão e por uma incisão abdominal com a outra. Indica-se a ressecção do útero prolapsado nos casos de longa posição em pé em que ocorreu necrose tecidual. Uma vez que o útero esteja em sua posição normal, antibióticos são colocados no mesmo, administra-se ocitocina e faz-se uma sutura de Caslick na vulva. As infusões de solução salina estéril e morna podem ajudar a evitar a recidiva (MANUAL MERCK DE VETERINARIA, 2006).

A fim de impedir possíveis recidivas, utilizam-se métodos de fechamento da vulva, tais como o de Flessa ou de Bühner, a vulva é untada com pomada de oxido de zinco adicionado a óleo de fígado de bacalhau. Após seis ou oito dias são retirados os pontos da sutura. 
SILVA, T.A. et al. Prolapso de cervix, vagina e útero em vacas - Revisão de Literatura. PUBVET, Londrina, V. 5, N. 27, Ed. 174, Art. 1176, 2011.

A sutura de Flessa pode ser feita com a agulha de flessa ou mesmo com a agulha em S, são feitos pontos de Wolf em dois ou três lugares, dependendo do tamanho da vulva, são utilizados captons para proteger a pele e evitar corte do fio. A sutura de Bühner é mais utilizada para vacas de vulva pequena, sendo feita com a agulha de Bühner ou com a agulha em $\mathrm{S}$, é feito um ponto grande de Wolf na vertical usando captons na parte superior e inferior da vulva, ficando perpendicular a comissura vulvar, a agulha não se aprofunda tanto quanto na sutura de Flessa (GRUNERT e BIRGEL, 1982)

O prognóstico depende do grau de lesão e de contaminação do útero. A reposição imediata de um útero limpo e minimamente traumatizado permite um prognóstico favorável. Não há tendência da afecção para recidivar nos partos subseqüentes. As complicações tendem a se desenvolver quando ocorrem laceração, necrose e infecção, ou quando se retarda o tratamento. (MANUAL MERCK DE VETERINARIA, 2006).

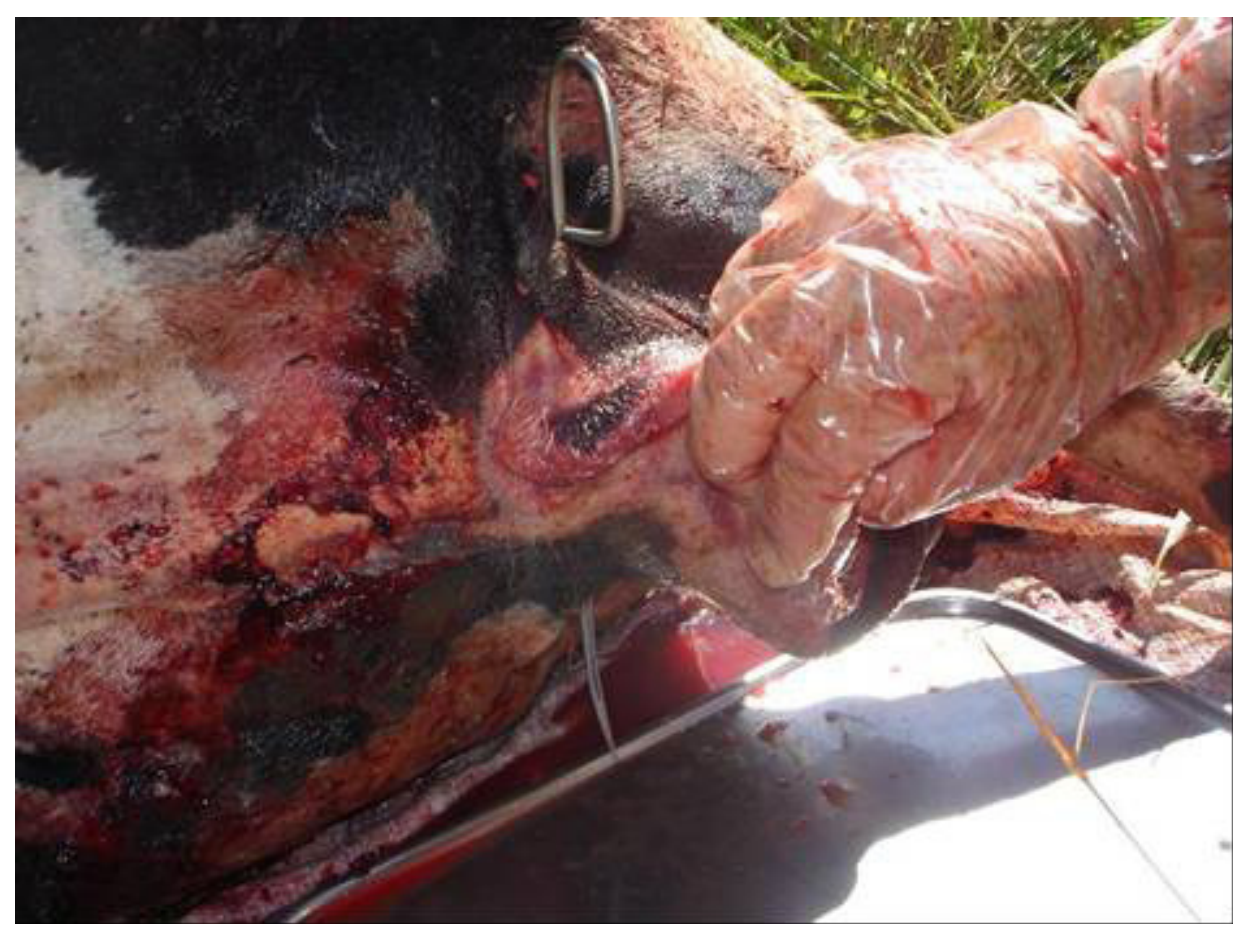

Figura 6: Realização da sutura de Flessa, com agulha de Flessa em uma vaca da raça Holandesa. 
SILVA, T.A. et al. Prolapso de cervix, vagina e útero em vacas - Revisão de Literatura. PUBVET, Londrina, V. 5, N. 27, Ed. 174, Art. 1176, 2011.

O choque, a hemorragia e o tromboembolismo são seqüelas comuns de um prolapso prolongado e requerem uma terapia de suporte agressiva.

Em alguns casos, a bexiga e os intestinos podem se prolapsar no útero evertido. Isso requer uma recolocação cuidadosa antes da recolocação do útero. Pode-se drenar a bexiga com um cateter ou uma agulha através da parede uterina. A elevação dos quartos posteriores e a pressão no útero ajudam na reposição da bexiga e dos intestinos.



Figura 7: Desenho esquemático da sutura de Caslick feita na vulva, de uma fêmea bovina, com fio cat-gute tipo C número 4 utilizando agulha curva cortante ponta triangular.

Fonte: Susan L. Fubini; Norm G. Ducharme (2004)

Pode-se tornar necessária a incisão do útero para se recolocarem esses órgãos. Na vaca, a amputação do útero severamente traumatizado ou necrótico pode ser o único meio de salvação do animal. O tratamento de suporte e a antibioticoterapia são indicados. 
SILVA, T.A. et al. Prolapso de cervix, vagina e útero em vacas - Revisão de Literatura. PUBVET, Londrina, V. 5, N. 27, Ed. 174, Art. 1176, 2011.

A eversão e o prolapso da vagina, com ou sem o prolapso da cervix, ocorrem em todas as espécies, mas são mais freqüentes nos bovinos e nos ovinos. Ocasionalmente, a bexiga pode estar contida na vagina prolapsada. A afeç̧ão geralmente ocorre nas fêmeas adultas no final da prenhez.

Os fatores predisponentes incluem o relaxamento e o aumento da mobilidade das estruturas de tecido mole no canal pélvico e no períneo à medida que o parto se aproxima e ao aumento da pressão intra-abdominal devido ao aumento do tamanho fetal, à gordura intra-abdominal ou à distensão do rúmen.

O Manual Merck de Veterinária (2006) cita que a afeç̧ão também pode ter um componente genético, já que freqüentemente é descrita como ocorrendo em algumas famílias e podem ocorrer em animais não prenhes jovens. No entanto, a maioria dos prolapsos ocorre nas vacas multíparas, o que sugere que os partos múltiplos predisponham a eversão. O relaxamento da vulva, da vagina e do tecido mole que a circunda no final da prenhez permite um aumento da mobilidade do trato reprodutivo caudal.

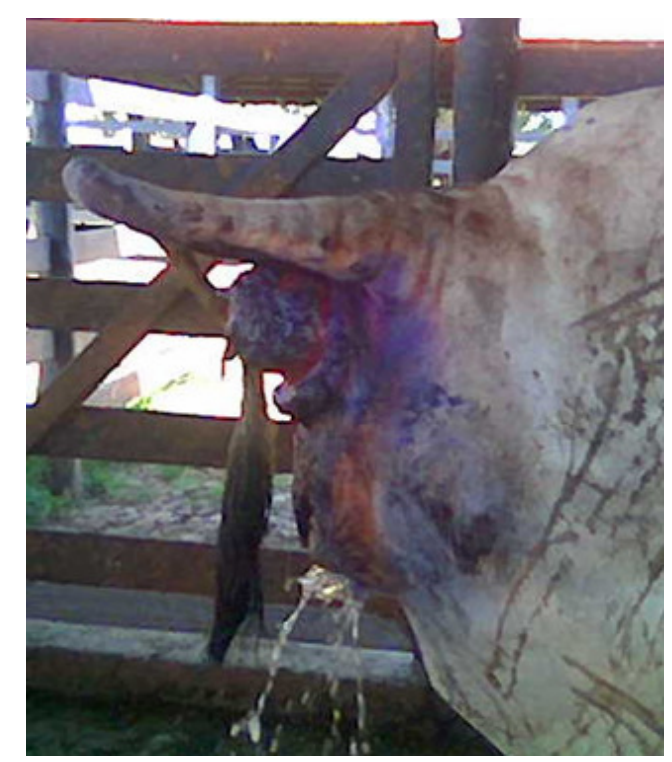

Figura 8: Inicio do prolapso de vagina e reto na gestação avançada de uma vaca da raça nelore com quatro anos de idade. 
SILVA, T.A. et al. Prolapso de cervix, vagina e útero em vacas - Revisão de Literatura. PUBVET, Londrina, V. 5, N. 27, Ed. 174, Art. 1176, 2011.

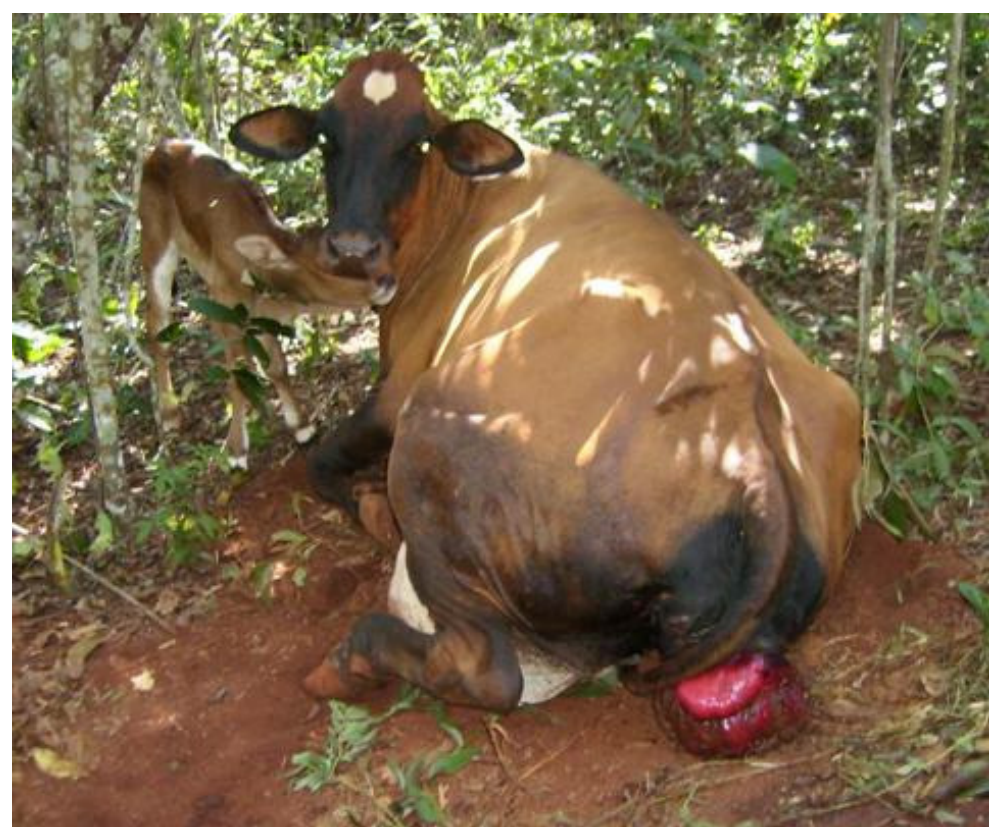

Figura 9: Prolapso de vagina e cervix acompanhado do quadro de hipocalcemia em uma vaca de cinco anos de raça mestiça com aptidão leiteira.

Quando o animal se deita, a gravidade e o aumento da pressão intraabdominal evertem temporariamente a vagina através da vulva. Uma irritação e um inchaço da mucosa exposta se seguem a uma eversão repetida, isto resulta em uma distensão e ocorre um prolapso. O assoalho da vagina se prolapsa primeiro e as eversões repetidas podem resultar em um divertículo de um ou ambos os lados da vagina.

A cervix se prolapsa ocasionalmente através da vulva. A abertura cervical externa pode ficar aumentada e eritematosa; no entanto, geralmente não se interrompe a prenhez.

Pode-se obstruir a uretra e impedir a miç̧ão, o que pode levar à ruptura da bexiga. Se não for tratada, resulta em uremia, estase vascular, necrose e infecção da vagina, e finalmente, morte. 
SILVA, T.A. et al. Prolapso de cervix, vagina e útero em vacas - Revisão de Literatura. PUBVET, Londrina, V. 5, N. 27, Ed. 174, Art. 1176, 2011.

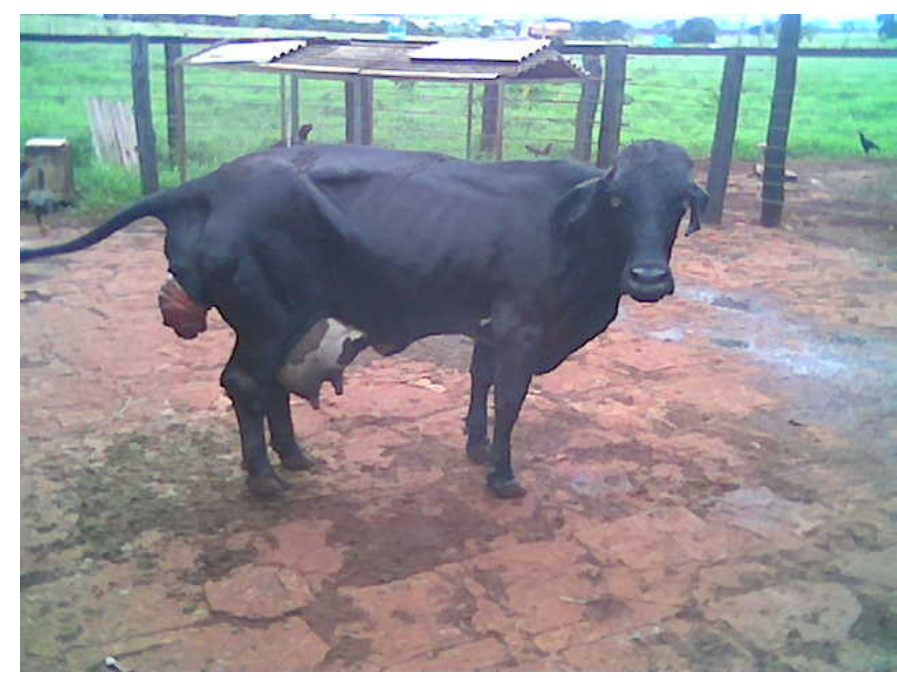

Figura 10: Vaca anã de quatro anos de idade com prolapso de vagina e cervix pós-parto.

Não se devem acasalar as famílias predispostas à afecção e os animais que já tenham sido afetados anteriormente. As práticas de alimentação devem ser avaliadas para se assegurar que os animais estejam ganhando peso no último trimestre mas não estejam sendo superalimentados, e que se tenham eliminado as fontes estrogênicas.

Os animais devem ser mantidos no nível do chão, durante o final da prenhez. Os métodos para impedir um prolapso intermitente no final da prenhez incluem várias técnicas de sutura ou o uso de um dispositivo de retenção (retentor de prolapso de Johnson).

A ressecção de uma parte da mucosa da parede vaginal, a fixação da parede cervical ou vaginal e a anestesia epidural com a utilização de álcool para impedir a distensão constituem outras soluções a longo prazo para o prolapso recidivante.

Após a administração do anestésico epidural, lava-se o órgão com sabão e água e enxágua-se completamente; se necessário, esvazia-se a bexiga; a congestão e o edema são reduzidos por meio da aplicação de uma pressão gentil; recoloca-se a vagina e aplica-se um antibiótico tópico. 
SILVA, T.A. et al. Prolapso de cervix, vagina e útero em vacas - Revisão de Literatura. PUBVET, Londrina, V. 5, N. 27, Ed. 174, Art. 1176, 2011.

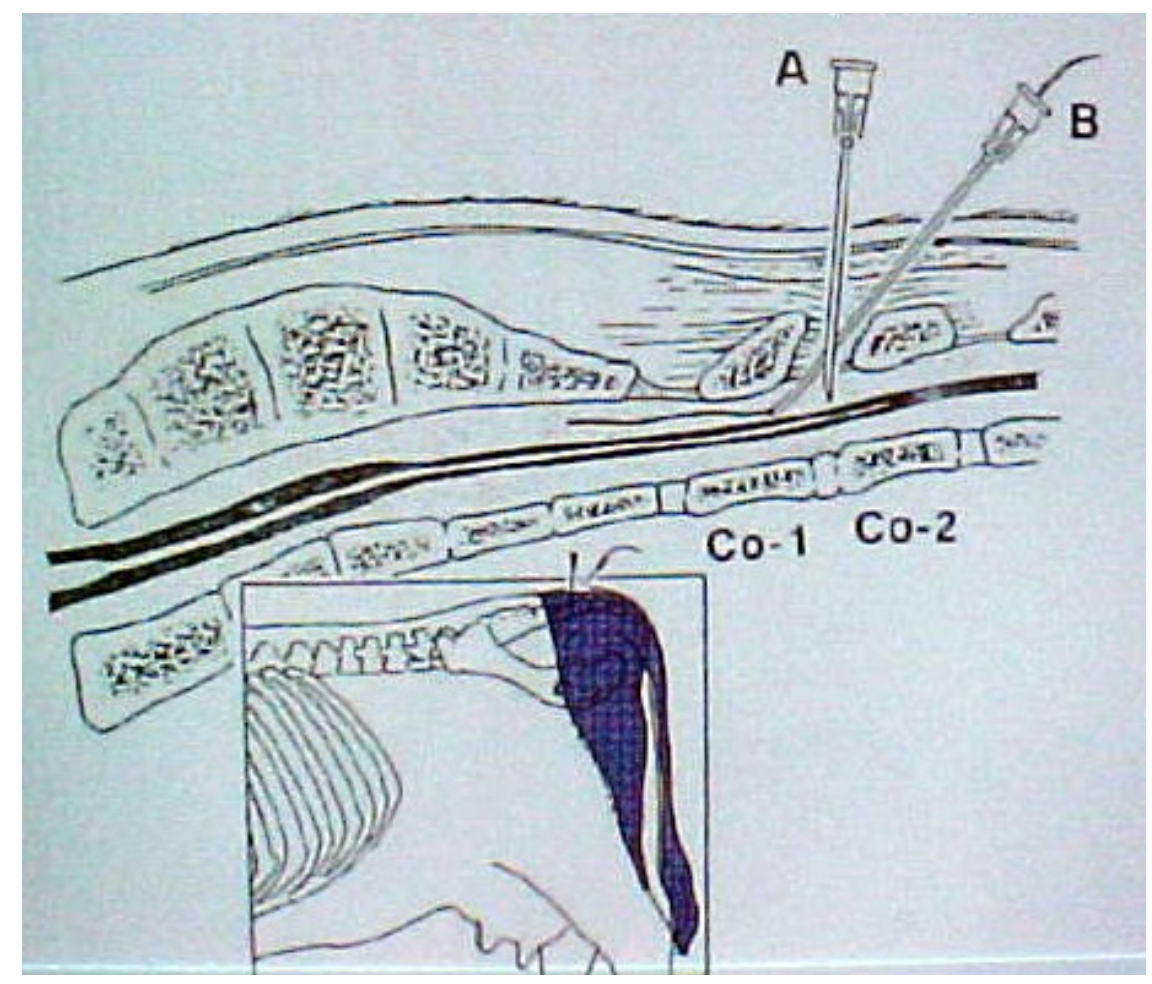

Figura 11: Demonstração da anestesia epidural baixa em bovinos, realizada entre as vértebras coxígeas 1 e 2 .

Então deve ser retida a vagina na posição. Somente o último procedimento apresenta uma dificuldade séria. A irritação da mucosa vaginal resulta em um tenesmo extremo, e os dispositivos de retenção devem ser fortes para impedir a recidiva.

A retenção é conseguida temporária ou permanente por meio de vários métodos de sutura da vulva. Grampos metálicos de prolapso com botões pesados ou dispositivos semelhantes também têm sido utilizados. Esses dispositivos são removidos durante o primeiro estágio do parto (MANUAL MERCK DE VETERINARIA, 2006.).

Como uma complicação rara do prolapso de vagina durante a realização do parto, foram apresentados alguns casos de fistulas vesico-vaginal, com derrame de urina nos tecidos circundantes a pelve, formação de abscessos urinários, peritonite séptica e consecutiva morte do animal (VATTI, 1962). 
SILVA, T.A. et al. Prolapso de cervix, vagina e útero em vacas - Revisão de Literatura. PUBVET, Londrina, V. 5, N. 27, Ed. 174, Art. 1176, 2011.

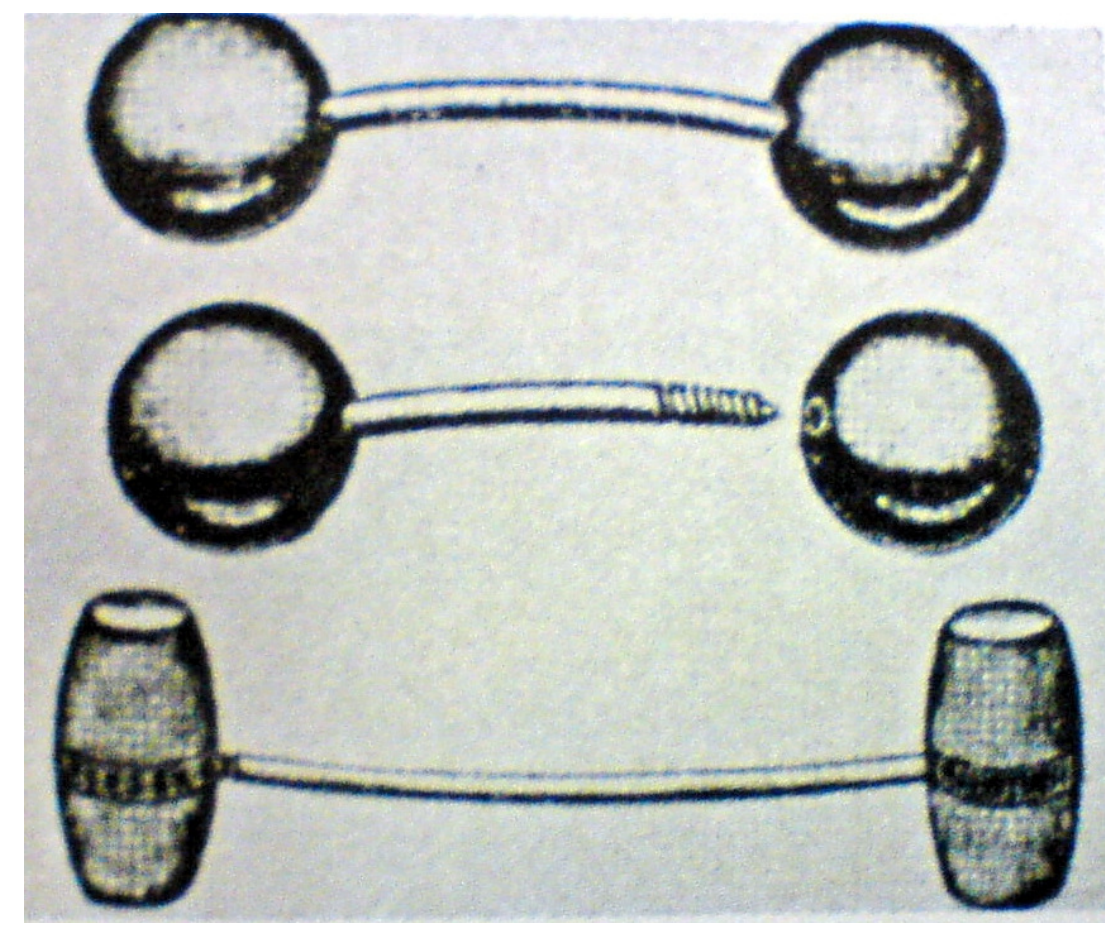

Figura 12: Grampos metálicos de Flessa .

Fonte: Catalogo de Hauptner, GIUSEPPE VATTI (1962).

\section{Estudo da incidência de prolapsos}

Nas variações de cada caso de prolapso o método de tratamento dependera do tempo de exposição das estruturas prolapsadas e da gravidade das lesões (ROBERTS, 1979).

Os prolapsos de vagina, cervix e útero são responsáveis por grandes perdas econômicas e desequilíbrio na eficiência reprodutiva de bovinos de leite e corte (BARTOLOMEU et al 1997 e MARQUES et al 1991).

Segundo Shukla \& Pareth (1987) a literatura relata de modo geral uma incidência de $3,35 \%$ a 5,36\%, dependendo da raça explorada ou manejo realizado.A causa exata dos prolapsos não foi definida, porem é comum acreditar que vários fatores desempenham um papel determinado.

Bovinos de corte, particularmente da raça Hereford, são afetados com mais freqüência, Woodward e Queensberry (1956) registraram 1,1 por cento de prolapsos vaginais em 7859 bovinos prenhes da raça Hereford nos Estados 
SILVA, T.A. et al. Prolapso de cervix, vagina e útero em vacas - Revisão de Literatura. PUBVET, Londrina, V. 5, N. 27, Ed. 174, Art. 1176, 2011.

Unidos, isto tem sugerido que nestes animais o arcabouço anatômico do aparelho genital é menos eficiente que em outros.



Figura 13: Fêmea de cinco anos da raça Hereford.

Fonte: www.nebraskaphotos.com

Uma deposição excessiva de gordura no tecido perivaginal e o relaxamento dos ligamentos da vagina podem aumentar a mobilidade da mesma (GEOFFREY, 1979).

Estudaram-se os níveis séricos de cálcio, fósforo e magnésio de 54 vacas, provenientes de diversos municípios do Estado de São Paulo.

O grupo um foi constituído por 27 vacas, sendo seis com prolapso uterino e 21 com prolapso cervico-vaginal, e o grupo dois (testemunha) com igual número de vacas clinicamente sadias.

O grupo um apresentou níveis séricos de cálcio menores do que o testemunha $(P<0,01)$, sendo os níveis mais baixos verificados em vacas com prolapso uterino. 
SILVA, T.A. et al. Prolapso de cervix, vagina e útero em vacas - Revisão de Literatura. PUBVET, Londrina, V. 5, N. 27, Ed. 174, Art. 1176, 2011.

Então se observou diferença nos níveis séricos de fósforo entre os dois grupos. Vacas com prolapso apresentaram teor sérico médio de magnésio inferior às do grupo testemunha $(P<0,01)$.

Os resultados parecem indicar que a hipocalcemia pode ser um fator etiológico envolvido no desencadeamento de prolapsos em vacas durante o puerpério (Marques, L. C; Marques, J. A; Peiró, J. R; Oliveira, J. A; Mendes, L. C. N. 1996).

Em outro trabalho realizado em Davis, Califórnia um estudo de 12 meses de duração para determinar os padrões de prolapso uterino e fatores associados à sobrevivência pós tratamento, foi empreendido por nove veterinários que trabalham com gado leiteiro. De 220000 vacas em produção visitadas pelos veterinários, $200(0.09 \%)$ desenvolveram prolapso (155/169 com dados) a maioria nas primeiras 24 horas de trabalho de parto. A maioria das vacas (130/200) tiveram prolapso durante os meses de outono e inverno e assistência veterinária foi requerida em 47 de 200 partos que resultaram em prolapso.

Dobson (2002), contendo 90 casos de prolapso uterino onde havia uma predisposição em novilhas de gado de corte e vacas velhas. $O$ índice de mortalidade foi de $20 \%$ e o choque foi a causa comum de morte entre os animais.

Em um estudo no Reino Unido, de 40 vacas que se recuperaram de prolapso uterino, apresentaram á inseminação artificial uma taxa de infertilidade de $25 \%$ em relação aos $5.5 \%$ do grupo controle.( DOBSON, 2002)

Milne (1954) e Knox (1952) expressaram a opinião que os prolapsos são mais comuns entre animais Herefords que em outras raças de animais de corte. Uma incidência variável entre raças sugere diferenças de herdabilidade para esta característica.

Possíveis diferenças, na ocorrência do prolapso entre as áreas e os anos, na mesma raça, indica que a nutrição pode ter um efeito sobre a relação de incidência e grau de prolapsos. 
SILVA, T.A. et al. Prolapso de cervix, vagina e útero em vacas - Revisão de Literatura. PUBVET, Londrina, V. 5, N. 27, Ed. 174, Art. 1176, 2011.

Dados foram colhidos de 1935 a 1954 nos Estados Unidos e dados suplementares vieram de Montana, Canadá os quais foram obtidos no período de 1943 á 1954 (Tabela 2). Durante esse período houve um total de 93 casos de prolapso de 7859 nascimentos, ou uma incidência de 1,2\%. Destes 93 casos, 71 foram de prolapso vaginal e 22 de prolapso uterino. Todos os animais que sobreviveram ao prolapso foram descartados. A perda de vacas resultou em $68 \%$ de casos de prolapso uterino e $18 \%$ de casos de prolapso vaginal. Também observado no estudo, um outro fator importante, foi a idade em que acontecia os casos de prolapso, mas havia uma tendência em direção a redução da incidência após o primeiro parto e um aumento da incidência, após as vacas chegarem a uma idade de sete anos (Tabela 3 ).

Tabela 2: Incidência de prolapsos durante o período de estudo que vai do ano de 1935 a 1954.Obtidos nos Estados Unidos e dados suplementares vindos de Montana, Canadá.

\begin{tabular}{lccc}
\hline ANO & NUMERO DE & NUMERO DE CASOS & PORCENTAGEM \\
& NASCIMENTOS & DE PROLAPSO & DA INCIDENCIA \\
\hline 1935 & 269 & 0 & 0.0 \\
1936 & 245 & 0 & 0.0 \\
1937 & 146 & 2 & 1.4 \\
1938 & 261 & 4 & 1.5 \\
1939 & 220 & 0 & 0.0 \\
1940 & 237 & 0 & 0.0 \\
1941 & 326 & 1 & 0.3 \\
1942 & 366 & 1 & 0.3 \\
1943 & 401 & 1 & 0.2 \\
1944 & 437 & 0 & 0.0 \\
1945 & 438 & 9 & 2.1 \\
1946 & 461 & 4 & 0.9 \\
1947 & 481 & 8 & 1.7 \\
1948 & 522 & 13 & 2.5 \\
1949 & 410 & 9 & 2.2 \\
1950 & 410 & 11 & 2.7 \\
1951 & 555 & 8 & 1.4 \\
1952 & 597 & 9 & 1.5 \\
1953 & 559 & 6 & 1.1 \\
1954 & 518 & 7 & 1.4 \\
\hline TOTAL & 7859 & 93 & $\ldots$ \\
PROPORCÃO & $\ldots$ & $\ldots$ & 1.2 \\
\hline
\end{tabular}

Fonte: R. R. Woodward; J. R. Quesenberry (1956) 
SILVA, T.A. et al. Prolapso de cervix, vagina e útero em vacas - Revisão de Literatura. PUBVET, Londrina, V. 5, N. 27, Ed. 174, Art. 1176, 2011.

Tabela 3: Incidência de casos de prolapsos por idade, em anos.

\begin{tabular}{lrrrrrrrr}
\hline DADE DA VACA & 3 & 4 & 5 & 6 & 7 & 8 & 9 & 10 \\
NUMERO DE NASCIMENTOS & & & & & & & & \\
NUMERO DE CASOS DE PROLAPSO & 1779 & 1502 & 1258 & 1036 & 802 & 618 & 480 & 312 \\
PORCENTAGEM DA INCDENCIA & 28 & 12 & 14 & 8 & 11 & 8 & 6 & 6 \\
\hline
\end{tabular}

Fonte:R. R. Woodward; J. R. Quesenberry (1956)

Foi realizado um estudo interessante com dados coletados na Estação de pesquisa Livestock e Range da cidade de Miles em Montana no Canadá, correlaciona efeitos da incisão na cesariana, anexos fetais retidos, prolapsos e uma subseqüente fertilidade das vacas de corte, durante o período que vai de 1963 a 1967. Neste trabalho a atenção será voltada somente á observação dos dados sobre prolapso. Um total de 153 partos foram associados com prolapso do trato reprodutivo incluindo $124(81.0 \%)$, prolapsos vaginais e $29(19,0 \%)$ prolapsos uterinos. $O$ índice de prenhez de todas as matrizes que sofreram prolapso foi mais baixo $(P<.01)$ que o índice da raça em questão. $O$ índice de prenhez entre as matrizes primípara e multíparas foi de $28.0 \%$ e $57,9 \%$ respectivamente. $O$ prolapso uterino resultou em uma diminuição significativa nos índices de prenhez das matrizes. O resultado observado foi semelhante ao obtido por Woodward e Quesenberry (1956), uma incidência de 1,2 \%, com dados do mesmo rebanho.

A media do peso ao nascer foi mais alto para bezerros machos nascidos de matrizes multíparas que pariram e tiveram prolapso. $O$ peso ao nascer foi despresivelmente mais baixo, que a media contemporânea, para bezerros nascido de vacas primiparas.

Nascimentos envolvendo bezerros machos contaram para $58,8 \%$ de todos os casos de prolapso ( $P<.05$ ), machos estiveram envolvidos em $58,3 \%$ dos prolapsos vaginais e em $60,7 \%$ dos prolapsos uterinos. 
SILVA, T.A. et al. Prolapso de cervix, vagina e útero em vacas - Revisão de Literatura. PUBVET, Londrina, V. 5, N. 27, Ed. 174, Art. 1176, 2011.

Os índices de sobrevivência dessas matrizes, em questão, que sofreram prolapso e dos bezerros que nasceram destas, foi respectivamente, $28.0 \%$ e $57,9 \%$.

Tabela 4: Media de peso ao nascimento, de bezerros machos e fêmeas, em partos que ocorreram prolapso.

\begin{tabular}{|c|c|c|c|c|c|c|c|c|c|c|c|c|}
\hline \multirow[b]{3}{*}{ PROLAPSO } & \multicolumn{4}{|c|}{$\begin{array}{l}\text { MATRIZES PRIMIPARAS } \\
\text { DE } 2 \text { ANOS DE IDADE } \\
\text { (PESO AO NASCER) }\end{array}$} & \multicolumn{4}{|c|}{$\begin{array}{l}\text { MATRIZES PRIMIPARAS } \\
\text { DE } 3 \text { ANOS DE IDADE } \\
\text { (PESO AO NASCER) }\end{array}$} & \multicolumn{4}{|c|}{$\begin{array}{l}\text { MATREES } \\
\text { MULTIPARAS } \\
\text { (PESO AO NASCER) }\end{array}$} \\
\hline & \multicolumn{2}{|c|}{ MACHO } & \multicolumn{2}{|c|}{ FEMEA } & \multicolumn{2}{|c|}{$\mathrm{MACHO}$} & \multicolumn{2}{|c|}{ FEMEA } & \multicolumn{2}{|c|}{ MACHO } & \multicolumn{2}{|c|}{ FEMEA } \\
\hline & No. & $\mathrm{Kg}$. & No. & $\mathrm{Kg}$. & No. & $\mathrm{Kg}$. & No. & $\mathrm{Kg}$. & No. & $\mathrm{Kg}$. & No. & $\mathrm{Kg}$. \\
\hline VAGINAL & 17 & 34,8 & 10 & 32,5 & 15 & 36,8 & 6 & 35,7 & 38 & 39,3 & 34 & 38,3 \\
\hline UTERINO & 6 & 32,2 & 7 & 31,9 & 7 & 38,2 & 3 & 35,9 & 4 & 41.5 & 1 & 39.9 \\
\hline
\end{tabular}

Fonte: D.J. Patterson; R.A. Bellows; P.J. Burffening. (1981)

\section{Conclusão}

Conclui-se que o número de informações literárias á cerca de dados epidemiológicos, prognósticos, perdas econômicas e etiologia dos prolapsos vaginais e uterinos são poucos.

Em geral o prognostico é favorável, mas sempre subordinado ao estado do órgão. Nos casos graves, quando o animal apresenta lesões severas tais como necrose e trombose, o prognostico torna-se desfavorável, podendo ocorrer a morte logo após a redução do prolapso.

Um autor relata que taxa de mortalidade em 25 animais tratados de prolapso genital foi de $20 \%$, enquanto que outro, cita que a taxa de mortalidade após tratamento foi $18 \%$.

A maioria dos animais tratados com reposição mecânica dos órgãos e sutura de vulva torna-se estéreis após prolapso vaginal ou uterino, mostrando cervicites crônicas, com índice de reincidência da afecção em 34,88\% dos animais.

Os prolapsos vaginal e cervical se repetirão invariavelmente e se tornarão mais graves durante as gestações posteriores. 
SILVA, T.A. et al. Prolapso de cervix, vagina e útero em vacas - Revisão de Literatura. PUBVET, Londrina, V. 5, N. 27, Ed. 174, Art. 1176, 2011.

Alguns autores citam que, a raça Hereford, é a mais afetada e com maior freqüência, registraram 1,1 por cento de prolapsos vaginais em 7859 bovinos prenhes da raça Hereford nos Estados Unidos, isto tem sugerido que nestes animais o arcabouço anatômico do aparelho genital é menos eficiente que em outros.

A idade da vaca e a sua ordem de parição, juntamente com o sexo e peso do feto ao nascer são fatores importantes nos casos de prolapso.

Com o conhecimento técnico e estas informações se pode ter um controle eficaz e manejo adequado dos animais, maximizando produção evitando perdas econômicas, morte de animais com alto valor zootécnico e descartes de animais de alta produtividade dos rebanhos.

\section{Referências}

ANUALPEC 2004. Anuário da pecuária brasileira. Editora FNP (2004). p 1-386. Disponível em: <http://www.fnp.com.br/.> Acesso em: 30 jun. 2004.

ANUALPeC 2008; Anuário da Pecuária Brasileira. São Paulo:Agro FPN, instituto ifPN (2008), p14-21.

Anuário DBO 2008; DBO Editores Associados Ltda, janeiro 2008. p. 18-23.

BARTOLOMEU C.C.; SOARES P.C.; MACHADO E.H.L.; ALENCAR S.P. Incidência de prolapso vaginal e uterino em vacas atendidas na clinica de bovinos, Campus Garanhuns. Revista Brasileira de Reprodução Animal, v. 21, n. 2, p. 177 - 180, 1997.

FUBUNI S. L.; DUCHARME N. G.; Farm Animal Surgery. USA: ed. Saunders 2004. 607p.

GARDNER I.A.; REYNOLDS J.P.; RISCOC.A.; HIRD D.W.; Patterns of uterine prolapse in dairy cows and prognosis after treatment. Department of Epidemiology and Preventive Medicine, School of Veterinary Medicine, University of California, Davis 95616. J. Am. Vet. Med. Assoc. 1990 Oct $15 ; 197(8): 1021-4$.

GEOFFREY H. A. Prolapso vaginal. Reprodução e obstetrícia em veterinária. Rio de janeiro; Editora Guanabara Koogan, (1979). p 114-120

GURNERT E.; BIRGEL E. H.; Obstetrícia veterinaria. Porto Alegre; Editora Sulina, (1982). $323 \mathrm{p}$.

KAHN C. A; Line C.; AIELLO S. E.; The Merck Veterinary Manual 9th Edition, 2006. Disponível em :< http://www.merckvetmanual.com/mvm/index.jsp>. Acesso em 28 maio.

2008.

KNOX J. H. Breeds of beef cattle for the southwest. Tour. Range Mgt (1952). 5:115. MILNE, E. J. EfGcaine-an aid in the treatment of bovine vaginal prolapse. Jour. Am. Vet. Med. Assn (1954). 124:108. 
MARQUES L.C.; JUNIOR D.C.; MARQUES J.A. Prolapso cervico-vaginal em bovinos; níveis séricos de cálcio, fósforo e magnésio. Clinica Veterinária De Jaboticabal, v. 5, n. 1, p. 6 8, 1991.

MARQUES L. C; MARQUES J. A; PEIRÓ J. R; OLIVEIRA J. A; MENDES L. C. N. Níveis séricos de cálcio, fósforo e magnésio em vacas com prolapso cervico-vaginal ou uterino / Calcium, phosphate and magnesium serum levels in cows with cervical-vaginal or uterine prolapse; Arq. bras. méd. vet. zootec;48(2):165-73, abr. 1996.

MAURICIO A. ELZO; Arcádio de los reyes borjas. Perspectivas da avaliação genética multiracial em bovinos no Brasil, 22 de dezembro de 2004.

MILNE E. J.; Efccaine-an aid in the treatment of bovine vaginal. Jour. Am. Vet. Med. ASSN. 124:108. (1954)

MURPHY A.M.; DOBSON H.; Predisposition, subsequent fertility, and mortality of cows with uterine prolapse. Vet. Rec. 2002; v151:p 733-735.

PANDIT R.K.; GRUPTA S.K.; PATTABI S.R. Clinical study on prolapse of vagina and uterus in buffaloes. Indian Veterinary Journal, v.59, p.975-980, 1982.

PATTERSON D.J.; BELLOWS R.A.; BURFENING P.J; Effects of Cesarean Section, Retained Placenta and Vaginal or Uterine Prolapse on Subsequent Fertility in Beef Cattle. Journal of animal science. J. Anim Sci 1981.v 53, p 961-921

ROBERTS S.J. Obstetrícia Veterinária y Patologia de la Reproducion. Buenos Aires: Hemisfério Sur. 1979. p. 248-257.

SHUKLA S.P.; PARECH H.K.B. Utero-vaginal prolapse in Gir cows and their exotic crosses. Indian Veterinary Journal, v. 64, p. 1050 - 1052, 1987.

VATTI G.; Lesiones y Complicaciones Consecutivas Al Parto. In: Ginecologia y Obstetrícia Veterinárias, 1962. P. $443-451$.

WOODWARD R. R.; QUENSENBERRY J. R.; A Study of Vaginal and Uterine Prolapse in Hereford Cattle. J Anim Sci 1956. 15:119-124. Disponível em: < http://jas.fass.org > Acesso em 28 de maio de 2008. 